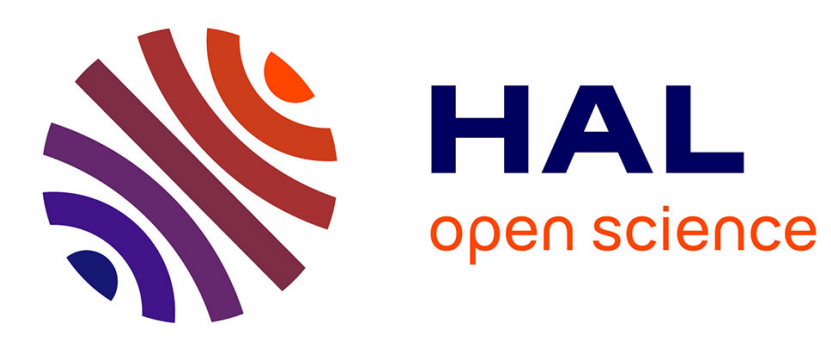

\title{
Les barrières à la mise en ouvre du crowdsourcing pour innover
}

\author{
Émilie Ruiz, Sébastien Brion, Guy Parmentier
}

\section{To cite this version:}

Émilie Ruiz, Sébastien Brion, Guy Parmentier. Les barrières à la mise en œuvre du crowdsourcing pour innover. Revue Française de Gestion, 2017, 43 (263), pp.121 - 140. 10.3166/rfg.2016.00105 . halshs-01630442

\section{HAL Id: halshs-01630442 \\ https://shs.hal.science/halshs-01630442}

Submitted on 11 Nov 2017

HAL is a multi-disciplinary open access archive for the deposit and dissemination of scientific research documents, whether they are published or not. The documents may come from teaching and research institutions in France or abroad, or from public or private research centers.
L'archive ouverte pluridisciplinaire HAL, est destinée au dépôt et à la diffusion de documents scientifiques de niveau recherche, publiés ou non, émanant des établissements d'enseignement et de recherche français ou étrangers, des laboratoires publics ou privés. 


\title{
Les barrières à la mise en œuvre du crowdsourcing pour innover
}

\author{
Emilie Ruiz \\ IREGE, Université Savoie Mont Blanc \\ emilie.ruiz@univ-smb.fr \\ Sébastien Brion \\ CRETLOG, Aix-Marseille Univ. \\ Guy Parmentier \\ CERAG, Univ. Grenoble Alpes
}

\section{Résumé}

Le crowdsourcing (CS) consiste à externaliser une tâche auprès d'un vaste réseau d'individus via un appel ouvert, le plus souvent réalisé par Internet. Malgré des difficultés identifiées dans la littérature, peu de recherches se sont intéressées aux barrières à l'adoption du CS pour innover. Cet article propose de les identifier et d'étudier leur importance lors de la phase de mise en œuvre. À partir de cinq cas d'entreprises, nous identifions trois catégories de barrières qui influencent cette activité et soulignons l'effet des barrières organisationnelles, notamment sur les difficultés de coordination avec la foule et celles liées aux ajustements organisationnels nécessaires à l'intégration de l'activité.

Mots-clés : innovation ouverte, crowdsourcing pour innover, barrières à l'adoption, mise en œuvre.

\section{Barriers to crowdsourcing for innovation implementation}

\begin{abstract}
Crowdsourcing (CS) is the outsourcing of a task to a wide network of individuals through an open call on the Internet. Although extant literature has identified some of the difficulties associated with it, little is known about barriers to CS for innovation implementation. This article aims to identify these barriers and to study their influence during the implementation stage. From five study cases, we identify three categories of barriers that affect CS for innovation, and we highlight the importance of organizational barriers, specifically difficulties related to coordination with the crowd and the organizational adjustments required for the integration of CS for innovation.
\end{abstract}

Keywords: open innovation, crowdsourcing for innovation, barriers to adoption, implementation. 


\section{Les barrières à la mise en œuvre du crowdsourcing pour innover}

\section{INTRODUCTION}

Dans la perspective de l'innovation ouverte, les entreprises peuvent choisir de s'ouvrir à des partenaires extérieurs tels que leurs clients ou utilisateurs de leurs produits pour capter de nouvelles idées, connaissances ou technologies (Chesbrough, 2003). Busarovs (2011) identifie le crowdsourcing (CS) comme une activité pertinente permettant aux entreprises d'innover avec leurs clients et utilisateurs, et plus largement avec la foule. Pour une entreprise, le CS consiste à prendre une tâche généralement exécutée en interne et à l'externaliser à un vaste réseau d'individus par un appel ouvert (Howe, $2006: 15$ ).

La littérature portant sur le CS s'est attachée pour l'essentiel à définir le concept (Howe, 2006 ; Lebraty, 2009 ; Schenk et Guittard, 2012), établir diverses taxonomies (Howe, 2006 ; Schenk et Guittard, 2012) ou proposer des études de cas permettant de comprendre les étapes constitutives de cette activité (Boutigny et Renault, 2013). L'intérêt du CS a été largement souligné dans la littérature, tant au niveau financier, par la réduction de coûts (Lebraty, 2009 ; Pénin et Burger-Helmchen, 2012 ; Schenk et Guittard, 2012), qu'au niveau de l'acquisition de nouvelles ressources et compétences (Howe, 2006 ; Afuah et Tucci, 2012 ; Pénin et BurgerHelmchen, 2012 ; Di Guardo et Castriotta, 2013). En outre, la «gratuité » apparente de ce phénomène peut fortement inciter les entreprises à adopter cette nouvelle pratique.

Toutefois, en dépit de ces avantages, le CS se heurte à de nombreuses barrières, notamment lorsqu'il est dédié à l'innovation (CS de résolution de problèmes complexes et CS de tâches créatives, Schenk et Guittard, 2012). Dans la lignée des travaux sur l'adoption de nouvelles pratiques (Damanpour, 1991 ; Damanpour et Schneider, 2006), la littérature sur le CS met en évidence certaines difficultés lors de sa mise en œuvre. La perméabilité du CS avec l'environnement peut par exemple conduire à des risques d'opportunisme (Pénin et BurgerHelmchen, 2012), et la nature tacite des connaissances manipulées (von Hippel et Katz, 2002) 
peut freiner le processus d'innovation (Pénin et Burger-Helmchen, 2012). Toutefois, les travaux sur les barrières à l'adoption de cette pratique sont rares. Dans le domaine particulier des challenges technologiques, Lüttgens et al. (2014) ont étudié les barrières à l'adoption du $\mathrm{CS}$, mais cette recherche se limite au CS de résolution de problèmes complexes et ne représente donc qu'une partie des pratiques de CS pour innover. Les auteurs identifient une série de barrières internes, notamment liées aux ressources humaines, comme freinant l'adoption d'une activité de CS de résolution de problèmes (manque d'implication de certaines fonctions, syndrome Not Invented Here etc.). Il apparaît essentiel d'étendre ces résultats à l'ensemble des activités de CS pour innover, notamment celle plus exigeante en terme de ressources (Pénin et Burger-Helmchen, 2012) : le CS d'activités créatives (Schenk et Guittard, 2012). L'objectif de cette recherche empirique est ainsi de répondre à la problématique suivante : quelles sont les barrières à la mise en ouvre du CS pour innover?

Pour y parvenir, nous développons une démarche exploratoire reposant sur cinq cas de CS pour innover qui portent sur la phase de mise en œuvre, considérée comme une phase critique de l'adoption de nouvelles pratiques (Damanpour et Schneider, 2006). Notre recherche identifie trois catégories de barrières (stratégiques, organisationnelles et cognitives) et souligne l'importance des barrières organisationnelles (notamment les difficultés de coordination avec la foule et d'intégration du CS à l'organisation). Nous mettons également en avant que les barrières ont un poids plus important sur le CS d'activités créatives.

Cet article est organisé en trois parties. La première propose une revue de la littérature dans laquelle nous définissons le concept de CS et les barrières à l'adoption de nouvelles pratiques, que nous mettons en perspective avec celles du CS identifiées dans la littérature. La deuxième partie présente la démarche méthodologique et les cas. Dans la troisième partie, les résultats nous permettent de mettre en évidence les principales barrières à la mise en œuvre du CS, 
selon les types de pratiques. Enfin, nous discutons des implications et limites de cette recherche.

\section{LES DÉTERMINANTS DU CROWDSOURCING}

\subsection{LE CROWDSOURCING : DEFINITION ET CONCEPT}

Le terme crowdsourcing a été popularisé par Howe (2006). Il s'agit de la contraction de crowd, la foule, et d'outsourcing, l'externalisation. Howe (2006) définit le crowdsourcing de la manière suivante: "crowdsourcing is the act of taking a job traditionally performed by a designated agent (usually an employee) and outsourcing it to an undefined, generally large group of people in the form of an open call» (Howe, 2006: 15). La littérature propose de nombreuses typologies de CS. Nous retenons celles plus récentes de Pénin et BurgerHelmchen (2012) ainsi que Schenk et Guittard (2012) qui débouchent sur trois types principaux. On trouve d'abord les activités routinières. Il s'agit d'activités basées sur des tâches simples et répétitives (Pénin et Burger-Helmchen, 2012), qui en s'agrégeant, vont permettre de créer de la valeur (Schenk et Guittard, 2012). Les autres types d'activités concernent plus directement l'innovation. Il s'agit d'une part du CS dont la finalité est de résoudre des problèmes complexes (Howe, 2006 ; Lebraty, 2009 ; Afuah et Tucci, 2012 ; Schenk et Guittard, 2012), et d'autre part du CS portant sur les activités créatives (Schenk et Guittard, 2012). Dans cet article, nous nous focalisons uniquement sur ces deux derniers types de CS qui appellent à une plus grande proximité entre la foule et les processus d'innovation et l'organisation de l'entreprise.

Le premier type de CS se réfère aux contributions de type résolution de problèmes. Schenk et Guittard (2012) parlent notamment de CS de «tâches complexes » lorsque la contribution attendue est la résolution de problèmes complexes ${ }^{1}$. Le second type de CS lié aux activités d'innovation identifié dans la littérature repose sur une démarche plus libre et plus ouverte,

\footnotetext{
${ }^{1}$ A titre d'exemple, la plateforme Innocentive met en relation des entreprises du secteur pharmaceutique et biomédical avec des experts dans le but de résoudre des problèmes spécifiques : http://www.innocentive.com/
} 
c'est-à-dire lorsque l'entreprise propose à la foule de participer au développement de nouveaux produits en générant de nouvelles idées ou en participant à des activités de cocréation. Schenk et Guittard (2012) parlent ici de CS de «tâches créatives ». Du point de vue organisationnel, l'entreprise fait entrer la foule dans son processus d'innovation via un appel ouvert $^{2}$. Compte tenu de la proximité avec le processus d'innovation de l'entreprise, ces types de CS peuvent poser plusieurs problèmes, notamment organisationnels, puisque les modèles d'apprentissage et l'organisation de l'entreprise peuvent être modifiés (Schlagwein et BjørnAndersen, 2014).

Tableau 1 : types de CS identifiés dans la littérature

\begin{tabular}{|l|l|l|l|}
\hline \multirow{2}{*}{} & \multicolumn{3}{|c|}{ Activités de crowdsourcing } \\
\hline Types de CS & \multicolumn{1}{|c|}{ CS d'activités créatives } & \multicolumn{1}{|c|}{$\begin{array}{c}\text { CS de résolution de } \\
\text { problèmes complexes }\end{array}$} & CS de tâches simples \\
\hline $\begin{array}{l}\text { Organisation } \\
\text { de l'activité }\end{array}$ & $\begin{array}{l}\text { La foule est « tirée » dans } \\
\text { l'organisation et le processus } \\
\text { d'innovation de l'entreprise. }\end{array}$ & $\begin{array}{l}\text { L'entreprise « pousse » un } \\
\text { problème précis et complexe } \\
\text { vers la foule. }\end{array}$ & $\begin{array}{l}\text { L'entreprise « externalise » un } \\
\text { problème ou une tâche simple } \\
\text { à la foule. }\end{array}$ \\
\hline $\begin{array}{l}\text { Modalités de } \\
\text { mise en } \\
\text { œuvre }\end{array}$ & $\begin{array}{l}\text { L'entreprise identifie une } \\
\text { tendance ou une } \\
\text { problématique pour laquelle } \\
\text { elle s'ouvre aux idées de la } \\
\text { foule. }\end{array}$ & $\begin{array}{l}\text { L'entreprise identifie en } \\
\text { amont un problème bien } \\
\text { spécifique et soumet un } \\
\text { cahier des charges limité et } \\
\text { précis à la foule. }\end{array}$ & $\begin{array}{l}\text { L'entreprise soumet } \\
\text { un problème simple ou une } \\
\text { tâche routinière qui requière } \\
\text { un grand volume de } \\
\text { contributions. }\end{array}$ \\
\hline $\begin{array}{l}\text { Compétences } \\
\text { recherchées }\end{array}$ & $\begin{array}{l}\text { Les activités de CS pour innover requièrent des compétences } \\
\text { rares et spécifiques de la part de la foule. }\end{array}$ & $\begin{array}{l}\text { Le CS de tâches simples ne } \\
\text { nécessite pas de compétences } \\
\text { particulières. }\end{array}$ \\
\hline $\begin{array}{l}\text { Modalités } \\
\text { relationnelles }\end{array}$ & $\begin{array}{l}\text { Interactions fortes avec la foule. La foule ciblée par } \\
\text { l'entreprise peut être de différents types (clients, utilisateurs, } \\
\text { passionnés, experts, communautés). }\end{array}$ & $\begin{array}{l}\text { Interactions faibles avec la } \\
\text { foule. La foule est ici } a \text { priori } \\
\text { anonyme et isolée. }\end{array}$ \\
\hline
\end{tabular}

\subsection{LES BARRIERES A LA MISE EN GEUVRE DU CS}

D’une manière générale, Damanpour (1991) définit l'adoption d'une nouvelle pratique comme un processus en trois étapes. Tout d'abord, l'entreprise prend conscience d'un besoin ou d'un problème et prend la décision d'adopter une nouvelle pratique (décision d'adopter). Puis, dans un second temps, elle met concrètement en œuvre cette pratique (mise en usage). Enfin, la dernière étape du processus d'adoption consiste à généraliser et pérenniser la

\footnotetext{
${ }^{2}$ A titre d'exemple, la plateforme Lego Ideas de la société Lego permet aux internautes de soumettre des idées de nouveaux produits ou de voter pour les idées proposées : https://ideas.lego.com/
} 
pratique au sein de l'entreprise (poursuite de l'usage). Dubouloz (2013) propose une synthèse de la littérature sur les barrières à l'adoption des nouvelles pratiques lean et identifie trois catégories de barrières : internes, externes et liées aux attributs du lean.

Les recherches portant sur les barrières à l'adoption des activités de CS sont rares et fragmentées. En s'inspirant de la théorie des frontières de la firme (Santos et Eisenhardt, 2005), Pénin et Burger-Helmchen (2012) indiquent que les activités de CS pour innover peuvent être influencées par quatre frontières organisationnelles : la frontière d'efficience (encadrement des actions de la foule), d'influence (capacité de maitrise des contributeurs), d'identité (culture de l'entreprise) et de compétence (intégration des connaissances de la foule). La frontière d'efficience concentre les aspects stratégiques, tant économiques (coûts de gouvernance et de transaction) que juridiques (droits de propriété). Le recours au CS fait ainsi l'objet d'un arbitrage quant à l'intérêt économique d'un engagement dans une telle démarche. L'aspect économique apparaît également comme une limite dans la mesure où le manque de ressources financières peut compromettre l'adoption d'une activité de CS (Lüttgens et al., 2014).

La question plus épineuse de la propriété intellectuelle peut aussi constituer un frein. La littérature (Di Gangi et Wasko, 2009 ; Di Guardo et Castriotta, 2013 ; Renault, 2014) a largement souligné les difficultés liées à cette question dans le cadre des activités de CS. A l'exception de Brabham (2008), peu d'auteurs affirment de manière explicite que les résultats des actions menées par la foule deviennent la propriété de l'entreprise. Ainsi, la foule peut revendiquer la propriété de l'innovation si elle souhaite en tirer profit ou si elle estime qu'elle n'a pas trouvé la réponse aux motivations attendues en participant à l'activité de CS (Di Guardo et Castriotta, 2013).

Si la démarche stratégique peut constituer une condition déterminante à l'initiation des processus de CS, sa mise en œuvre appelle des questionnements organisationnels. Dans le 
domaine des activités de CS de résolution de problèmes complexes, Lüttgens et al. (2014) ont récemment mis en avant une série de barrières organisationnelles intervenant sur l'ensemble des étapes de déroulement de challenges technologiques : rigidité des processus existants dans l'entreprise, syndrome $\mathrm{NIH}$, désintérêt des fonctions de l'entreprise et du top management et manque de communication entre les fonctions impliquées. Ces dimensions font écho aux frontières identitaire et d'influence identifiées par Pénin et Burger-Helmchen (2012) où les valeurs du dirigeant, l'implication des ressources humaines et la structure de l'entreprise vont jouer un rôle clé sur sa capacité organisationnelle à assumer une telle démarche.

Les barrières portant sur les finalités et l'organisation du CS ne sauraient constituer les seules limites à l'adoption de ces nouvelles pratiques. La question du contenu de ce qui est externalisé à la foule fait aussi débat. Lorsque le problème posé s'éloigne du domaine de connaissance de l'entreprise, le CS semble s'imposer comme une solution plus efficace que le recours à des acteurs considérés comme experts (Afuah et Tucci, 2012). Toutefois, cette « frontière de compétence » (Pénin et Burger-Helmchen, 2012) peut être à double tranchant et limiter la capacité de l'entreprise à intégrer la nouvelle connaissance proposée par la foule, notamment lorsque cette dernière est de nature tacite (von Hippel, 1994). La capacité d'absorption (ACAP), c'est-à-dire la capacité de l'entreprise «à reconnaitre la valeur d'une information nouvelle, à l'assimiler et à l'exploiter »(Cohen et Levinthal, 1990 : 128), peut ainsi être considérée comme un prérequis à tout appel ouvert. Le recours aux activités de résolution de problèmes à proximité de la base de connaissance de l'entreprise (local search) lui permettra sans doute de faciliter l'intégration des solutions, mais elles auront un effet limité sur les capacités d'exploration de l'entreprise (March, 1991). En définitive, bien que portant sur différentes activités de CS, la littérature nous permet de délimiter trois grandes catégories de barrières à l'adoption de ces nouvelles pratiques (cf. tableau 2).

Tableau 2 : synthèse des barrières identifiées dans la littérature 


\begin{tabular}{|c|l|l|}
\hline Types de barrières & \multicolumn{1}{|c|}{ Barrières } & \multicolumn{1}{c|}{ Détails } \\
\hline $\begin{array}{c}\text { Barrières } \\
\text { stratégiques et } \\
\text { économiques }\end{array}$ & Manque de ressources & $\begin{array}{l}\text { Peu de ressources financières allouées à l'activité. Diverses conséquences : } \\
\text { difficultés à maintenir l'activité, à la valoriser en interne, à attirer la foule. }\end{array}$ \\
\cline { 2 - 3 } & Propriété intellectuelle & $\begin{array}{l}\text { Cession de la propriété de la contribution à l'entreprise pas toujours claire } \\
\text { pouvant être à l'origine de conflits avec la foule. }\end{array}$ \\
\hline \multirow{2}{*}{$\begin{array}{c}\text { Barrières } \\
\text { organisationnelles }\end{array}$} & $\begin{array}{l}\text { Rigidité des processus } \\
\text { existants }\end{array}$ & $\begin{array}{l}\text { Processus organisationnels peu flexibles ne permettant pas d'intégrer } \\
\text { l'activité de CS. }\end{array}$ \\
\cline { 2 - 3 } & Identité de l'entreprise & $\begin{array}{l}\text { Valeurs du dirigeant et de l'entreprise pouvant être à l'encontre du } \\
\text { principe d'ouverture à la foule. }\end{array}$ \\
\cline { 2 - 3 } & $\begin{array}{l}\text { Manque d'implication } \\
\text { des fonctions }\end{array}$ & $\begin{array}{l}\text { Désintérêt de certains individus pour l'activité voire syndrome NIH (refus } \\
\text { d'intégrer toutes connaissances externes). }\end{array}$ \\
\hline Barrière cognitive & ACAP & $\begin{array}{l}\text { Difficultés à identifier, comprendre et exploiter les connaissances de la } \\
\text { foule, pouvant être éloignées de celles de l'entreprise. }\end{array}$ \\
\hline
\end{tabular}

La littérature portant sur les barrières à l'adoption d'une innovation (Damanpour et Schneider, 2006 ; Dubouloz, 2013) souligne l'importance de la phase de mise en œuvre, décrite par Damanpour et Schneider (2006) comme la phase la plus critique, cristallisant l'apparition de nombreuses barrières. Compte tenu du caractère relativement récent de cette activité innovante, quelques rares travaux (Di Gangi et Wasko, 2009 ; Thuan et al., 2015) se sont penchés sur les facteurs qui influencent la décision d'adopter une activité de CS. Toutefois, aucune recherche du même type n'a encore été proposée sur la phase de mise en œuvre, en dépit de son importance.

\section{DÉMARCHE MÉTHODOLOGIQUE ET CONTEXTE DE LA RECHERCHE}

La section suivante présente la démarche méthodologique. Le CS pour innover étant un phénomène relativement récent, nous justifions par la suite en quoi notre démarche qualitative s'avère pertinente (Miles et Huberman, 2003).

\subsection{METHODOLOGIE DE LA RECHERCHE : RECUEIL ET ANALYSE DES DONNEES}

Cette recherche qualitative propose cinq études de cas (Yin, 2013) réalisées au sein de différentes entreprises (cf. tableau 3). Le but est de mieux comprendre les difficultés que rencontrent ces entreprises lors de la mise en œuvre d'une activité de CS pour innover.

\section{DEMARCHE METHODOLOGIQUE}

Notre recherche repose sur des données primaires et secondaires, mobilisant ainsi de multiples sources empiriques (Yin, 2013). Nous avons mené 13 entretiens semi-directifs entre avril 
2014 et octobre 2015 d'une durée moyenne d'1h30, auprès des dirigeants ou responsables des activités de CS des cinq entreprises étudiées. Tous les entretiens ont été enregistrés et retranscrits. Le guide d'entretien nous a permis d'explorer différents thèmes relatifs au CS (organisation de l'entreprise, décision d'adopter l'activité, barrières rencontrées lors de la mise en œuvre et lors de la transition vers l'étape de pérennisation) par le biais de questions ouvertes, pour ne pas influencer les répondants.

Les cas ont été sélectionnés selon des critères de similarité et de variété afin de pouvoir observer des régularités dans le phénomène étudié (Yin, 2013), à savoir la mise en œuvre du CS pour innover. Les cinq cas ont tous pour points communs de mettre en œuvre des activités de CS pour innover et sont issus du secteur industriel. Ils présentent toutefois des points de divergences, tels que le type de CS pour innover (activités créatives ou résolution de problèmes complexes) et la taille des entreprises étudiées.

Le traitement des données a été réalisé au moyen d'un codage thématique ${ }^{3}$ avec le logiciel NVivo. Cette démarche nous a permis d'identifier, de classer et de hiérarchiser nos données et de les découper en unités d'analyse (Miles et Huberman, 2003), ici en catégories de barrières. Nous avons suivi une démarche abductive, en partant des barrières identifiées dans la littérature. Par ailleurs, d'autres sources secondaires ont été mobilisées pour identifier le contexte et l'environnement de chaque cas et compléter les données primaires : sites Internet, plateformes de CS, études produites par les entreprises etc.

\begin{tabular}{|c|c|c|c|c|c|}
\hline \multicolumn{5}{|c}{ Tableau 3-caractéristiques des cas } \\
\hline $\begin{array}{c}\text { Secteur } \\
\text { d'activité }\end{array}$ & Télécoms & B & C & D & E \\
\hline Taille & $\begin{array}{c}\text { Grande } \\
\text { entreprise }\end{array}$ & $\begin{array}{c}\text { Grande } \\
\text { entreprise }\end{array}$ & $\begin{array}{c}\text { Textile et } \\
\text { matériel sportif }\end{array}$ & $\begin{array}{c}\text { Matériel et } \\
\text { équipements } \\
\text { sportifs }\end{array}$ & $\begin{array}{c}\text { Petit } \\
\text { électroménager }\end{array}$ \\
\hline $\begin{array}{c}\text { Type de CS } \\
\text { pour } \\
\text { innover }\end{array}$ & $\begin{array}{c}\text { Problèmes } \\
\text { complexes } \\
\text { Date } \\
\text { d'adoption }\end{array}$ & $\begin{array}{c}\text { Problèmes } \\
\text { complexes et } \\
\text { créativité }\end{array}$ & Créativité & Créativité & $\begin{array}{c}\text { Grande } \\
\text { entreprise }\end{array}$ \\
\hline $\begin{array}{c}\text { Nombre } \\
\text { d'entretiens }\end{array}$ & 2008 & $\begin{array}{c}\text { Problèmes } \\
\text { créativité }\end{array}$ \\
\hline $\begin{array}{c}\text { Fonction des } \\
\text { répondants }\end{array}$ & $\begin{array}{c}\text { Co-responsables } \\
\text { de la plateforme }\end{array}$ & $\begin{array}{c}\text { Responsable et } \\
\text { ancien co- } \\
\text { responsable de } \\
\text { la plateforme }\end{array}$ & $\begin{array}{c}\text { Dirigeant } \\
\text { et } \\
\text { Community } \\
\text { manager }\end{array}$ & $\begin{array}{c}\text { Responsable de } \\
\text { l'innovation }\end{array}$ & $\begin{array}{c}\text { 2013 } \\
\text { d'innovation } \\
\text { et } \\
\text { Chargé de } \\
\text { 1'innovation } \\
\text { ouverte }\end{array}$ \\
\hline
\end{tabular}

\subsection{Presentation des CaS}

Entreprise A (secteur des télécoms - CS de résolution de problèmes complexes)

L'entreprise A est une grande entreprise française à rayonnement international, dans le secteur des télécommunications. Elle fait appel depuis 2008 à la foule via une plateforme en ligne gérée par deux responsables sur laquelle sont proposées différentes sessions liées aux

\footnotetext{
${ }^{3}$ Un échantillon de la grille de codage est disponible sur demande.
} 
technologies et offres numériques. Les participants sélectionnés échangent, donnent leurs avis ou testent de nouveaux services pour répondre à un problème précis rencontré par l'entreprise. Ils sont rétribués par des chèques cadeaux. Avec près de 6000 inscrits, la plateforme rencontre un franc succès auprès des participants, mais souffre d'une mauvaise visibilité en interne, noyée au milieu d'autres dispositifs d'innovation ouverte.

Entreprise B (secteur de l'énergie - CS de résolution de problèmes complexes et d'activités créatives)

L'entreprise B est une grande entreprise française étatique du secteur de l'énergie. Suite à un accord signé avec le gouvernement dans le but de renforcer l'innovation entre les grands groupes et les PME, l'entreprise B a lancé en 2013 sa plateforme de CS destinée aux PME, constituée de deux parties. La première vise à proposer des challenges et la seconde sélectionne des «candidatures spontanées ». Anciennement gérée par le département partenariat de la Direction de l'Innovation, la plateforme est aujourd'hui intégrée à un programme d'innovation plus vaste. Fin 2015, 17 challenges ont été lancés sur la plateforme et 260 candidatures spontanées ont été reçues, débouchant sur 15 contrats de codéveloppement effectifs. Malgré cela, la plateforme souffre aujourd'hui d'un positionnement interne fragilisé par un contexte difficile.

Entreprise C (secteur du textile sportif - CS d'activités créatives).

L'entreprise C est une PME installée en Rhône-Alpes qui compte une trentaine de salariés. Créée en 2000 par un ingénieur textile passionné de sport, elle développe, conçoit et commercialise des équipements et textiles sportifs innovants pour les sports dits outdoor. Elle intègre des idées et propositions d'utilisateurs dès la phase de conception des produits via une plateforme virtuelle. Suite à un vote, des études et des tests de faisabilité complémentaires, l'entreprise fabrique en série ces propositions émises par sa communauté. Un système de 
rétribution par points permet de récompenser les contributeurs. L'entreprise anime également sa communauté autour des différents sports sur lesquels portent ses produits.

Entreprise D (secteur du matériel et équipements sportifs - CS d'activités créatives)

L'entreprise D est une grande entreprise française présente à l'international dans le domaine de la conception, la fabrication et la distribution de matériel et équipements sportifs. Le groupe a lancé, via l'une de ses marques, un premier challenge en 2012 en partenariat avec une plateforme internationale spécialisée dans l'animation de communautés d'innovation. Les internautes ont été invités à publier leur projet et le jury de la marque a désigné les trois projets les plus pertinents. Ces projets ont été récompensés par une dotation financière en échange de cession des droits de propriété. Suite à ce challenge, le groupe a décidé de déployer une plateforme interne partagée avec toutes les activités du groupe.

Entreprise $\boldsymbol{E}$ (secteur de l'électroménager - CS de problèmes complexes et activités créatives)

L'entreprise E est un acteur majeur dans le domaine de l'électroménager, implanté sur plusieurs continents. Le groupe a mis en place en 2013 une plateforme de CS composée d'un module d'activités créatives et d'un module de challenges pour résoudre des problèmes techniques complexes. Le premier module a pour but de structurer la relation entre le groupe et les inventeurs spontanés qui contactent l'entreprise depuis de nombreuses années. Le second module permet de proposer des challenges auxquels les internautes peuvent s'inscrire pour tenter de résoudre des blocages techniques. En parallèle, l'entreprise recherche activement des experts sur les réseaux sociaux, avec pour objectif final l'émergence d'une communauté.

\section{L'INFLUENCE DES BARRIÈRES SUR LA MISE EN AEUVRE DU CROWDSOURCING POUR INNOVER}

Nos résultats identifient sept barrières à la mise en œuvre du CS pour innover, correspondant aux trois grandes catégories suggérées par la littérature : stratégiques, organisationnelles et 
cognitives. Le tableau 4 présente une synthèse de ces barrières selon leur nature et leur importance perçue. Dans les parties suivantes, nous présentons de manière détaillée chacune de ces barrières.

\subsection{LES BARRIERES STRATEGIQUES : REVELER DES INFORMATIONS AUX CONCURRENTS, UNE PREOCCUPATION DES GRANDES ENTREPRISES}

Lorsqu'elles adoptent une activité de CS pour innover, les entreprises font face à des barrières stratégiques qui proviennent pour l'essentiel du top management. Dans l'ensemble des cas étudiés, ce type de barrières semble ralentir la mise en œuvre du CS pour innover mais ne constitue pas pour autant une réelle barrière remettant en cause son adoption. Lorsque le top management valide l'engagement de l'entreprise dans une activité de CS pour innover, les équipes en charge de ces projets ne bénéficient généralement que d'un budget limité. L'aspect économique peut ainsi avoir pour effet d'allonger le temps d'adoption du fait de budgets restreints, poussant les équipes à s'adapter.

«On avait des budgets très limités et on faisait avec ce qu'on avait » (Entreprise E, chargé de l'innovation ouverte, janvier 2015).

Une autre préoccupation stratégique du top management concerne la propriété intellectuelle. Afin de ne pas se retrouver en conflit avec les contributeurs et ne pas porter préjudice à l'image de l'entreprise, il apparait que les équipes en charge des plateformes de CS semblent porter une attention particulière aux aspects juridiques lors de leurs interactions avec la foule.

"C'est une préoccupation : on ne doit pas être perçu comme l'espèce de grand groupe qui va encore piquer toute la propriété des autres. » (Entreprise B, responsable de la plateforme de CS, octobre 2015).

Il apparait toutefois que les responsables des plateformes ne considèrent pas véritablement cette dimension comme une barrière. Ils anticipent ainsi la question des droits de propriété en amont du processus, par exemple en contrepartie de rétributions, en l'indiquant dans le cahier des charges.

"Concernant les trois premiers prix, en étant rémunérés, les gagnants cèdent leurs droits sur leur création » (Entreprise D, responsable de l'innovation, mars 2014). 
Si les barrières liées à la propriété intellectuelle, relatives au cadre légal, peuvent être levées à l'aide de moyens juridiques, la révélation d'informations stratégiques sur Internet semble préoccuper plus particulièrement les acteurs des grandes entreprises. En effet, la mise en ligne d'un problème à résoudre ou la révélation des thématiques d'intérêt pour innover (CS d'activités créatives) est perçue comme un véritable dévoilement à la concurrence des axes stratégiques de l'entreprise. La fonction $\mathrm{R} \& \mathrm{D}$ est particulièrement préoccupée par ce problème, pouvant entraver la mise en œuvre du CS pour innover.

"On a des personnes en BU gênées par le manque de confidentialité, qui nous disent que ce serait embêtant que les concurrents en profitent ». (Entreprise E, responsable des processus d'innovation, janvier 2015).

Toutefois, là encore, il semble que des solutions existent pour lever cette barrière et rassurer quant à la révélation d'informations potentiellement stratégiques. L'entreprise C (PME) explique que dévoiler ses projets d'innovation à la foule, donc potentiellement à ses concurrents constitués pour l'essentiel de grandes entreprises, ne lui pose pas de problème. Selon l'entreprise, l'agilité et la rapidité de développement de ses équipes constituent la meilleure arme stratégique face au dévoilement.

"Par rapport aux concurrents, c'est celui qui va développer le plus vite qui aura l'avantage » (Entreprise C, Dirigeant, juin 2014).

Les grandes entreprises de notre échantillon ne sont pas de cet avis. Selon le niveau de confidentialité des informations divulguées sur Internet, elles auront recours à des plateformes spécialisées qui leur permettront de publier et de gérer le challenge de manière anonyme et ainsi contourner cette barrière.

"Si on veut être parfaitement confidentiels, on passe par NineSigma [...] c'est une alternative au manque de confidentialité en fonction du problème posé. » (Entreprise E, chargé de l'open innovation, janvier 2015).

Compte tenu du caractère nécessairement ouvert de l'activité de $\mathrm{CS}$, les barrières stratégiques attendues dans ce domaine semblent relativement peu influentes. De manière générale, qu'il 
s'agisse des aspects financiers, de la propriété intellectuelle ou de la révélation des informations stratégiques, des solutions existent pour atténuer leurs effets potentiellement négatifs sur la mise en œuvre du CS. En marge de ces points communs, une différence importante semble se dégager entre les cas. A la différence des cas A, B, D et E issus de grandes entreprises, la vision de la PME (C) vis-à-vis de la révélation des informations est marquante et conduit à un possible clivage selon la taille de l'entreprise.

\subsection{Difficultes D'INTEgRATiON ET DE COORDinAtion AVEC LA FOULE : DES BARRIERES ORGANISATIONNELLES PREDOMINANTES}

A la différence d'autres initiatives d'innovation ouverte, les activités de CS pour innover engagent l'entreprise avec des partenaires a priori non identifiés : la foule. La coordination avec la foule est perçue, pour plusieurs raisons, comme une barrière majeure à la mise en œuvre du CS pour innover. L'ouverture de l'entreprise à ces ressources nous amène à constater dans l'ensemble des cas étudiés une difficulté quant à la quantité et la qualité des contributions reçues.

"On se retrouve avec beaucoup d'idées. Il faut les classer, les lire [...] 300 idées en 10 mois, 1000 réponses en 3 jours » (Entreprise C, Dirigeant, juin 2014).

Il existe également des spécificités dans l'animation des relations avec la foule. Dans l'ensemble des cas, les entreprises font face à de nombreux coûts de coordination. Outre un grand nombre de contributions reçues dont il faut prendre connaissance, la foule attend de l'entreprise dans tous les cas étudiés une grande réactivité.

"Faire de l'open innovation, ça coûte de l'argent, ne serait-ce qu'en animation. [...] On a réalisé que le community manager seul ne pouvait pas assurer ce rôle parce qu'il fallait presque répondre en temps réel » (Entreprise D, responsable de l'innovation, mars 2014).

Cette sollicitation de la foule tant en termes de quantité de contributions reçues que de réactivité peut également entrainer des problèmes organisationnels à plus long terme. L'entreprise D a par exemple dû employer trois chefs de projets successifs et plusieurs stagiaires pour s'adapter au nombre de contributions reçues, à l'étendue du projet et pour 
animer et gérer la communauté, représentant un coût supplémentaire et des difficultés organisationnelles en matière de gestion des ressources humaines, compte tenu du turn over qu'a subi le projet.

Il est toutefois intéressant de préciser que la barrière liée à la coordination avec la foule est d'autant plus importante dans le cas du CS d'activités créatives. Dans le cas du CS de résolution de problèmes complexes, les relations avec la foule sont limitées. L'entreprise A organise par exemple des sessions temporellement définies, focalisées sur un cahier des charges précis. A l'inverse, pour le CS d'activités créatives, les processus sont moins structurés et plus longs : instauration d'une relation plus durable avec la foule, réponses aux sollicitations au fil de l'eau etc. L'entreprise B gère par exemple sur sa plateforme ces deux types de CS pour innover et son portail «candidatures spontanées » lui demande plus de ressources que son portail « réponse à challenges ».

"Quand c'est une candidature spontanée, il faut identifier quels partenaires on va mettre dans la boucle. Quand c'est un challenge, c'est déjà identifié dès le départ. » (Entreprise B, ancien co-responsable de la plateforme, octobre 2015).

Nos cas mettent en évidence une certaine méfiance liée à la culture de l'organisation à l'égard de ces démarches. Certaines entreprises conditionnent la survie du dispositif de CS à l'obtention de résultats tangibles. La culture du résultat à court terme de l'entreprise E peut freiner l'adoption du CS et conduire à une mise en œuvre par étapes, moins efficace.

"On va d'abord prouver que ça a un intérêt pour l'entreprise [...] c'est bien dans la culture $d u$ groupe, on avance à petits pas » (Entreprise E, chargé de l'innovation ouverte, janvier 2015).

Au sein de cette entreprise, les deux personnes chargées du développement de la plateforme sont régulièrement tenues de présenter l'état d'avancement des challenges (nombre de contributeurs, taux de soumissions pertinentes etc.) et de réaliser des rapports à destination du top management. Ces obligations prennent du temps et montrent que des efforts importants 
sont nécessaires pour maintenir le dispositif dans l'entreprise alors qu'ils ne contribuent pas directement à son développement.

D'autres types de freins, d'ordre sectoriel, peuvent également influencer négativement la mise en œuvre du CS. En évoluant dans un secteur traditionnellement voué au secret industriel, l'entreprise $\mathrm{B}$ a le plus grand mal à faire accepter le principe d'ouverture attaché au CS en interne, certains salariés ayant du mal à accepter que l'entreprise aille chercher des connaissances externes. Cela révèle ainsi une barrière culturelle liée au syndrome NIH.

"Nous on est une culture du secret, voire du secret défense. C'est difficile d'aller rechercher ce qui se passe ailleurs et de se dire que c'est peut-être une bonne idée. » (Entreprise B, responsable de la plateforme, octobre 2015).

D'autres barrières liées à la gestion interne du CS viennent s'ajouter au manque d'implication interne et aux aspects culturels. A l'exception de la PME C, dont le business model repose sur l'ouverture à la foule, la difficulté d'intégrer les projets de CS au reste de l'organisation apparaît dans tous les autres cas. Qu'elle soit dédiée ou intégrée aux équipes d'innovation, la position organisationnelle de l'équipe projet de CS semble poser problème.

«Après se pose la question de l'équipe et de l'organisation en interne pour suivre et animer le projet. Il est décidé de mettre en place une équipe en marge de l'organisation classique.» (Entreprise D, responsable de l'innovation, mars 2014).

Dans le cas de l'entreprise D, il est par exemple demandé aux acteurs du projet de ne pas prendre de temps sur leur temps de travail «normal». Les équipes gèrent l'activité de CS en même temps que d'autres responsabilités, en temps masqué, ce qui rend difficile son intégration et limite sa visibilité. A l'inverse, même lorsque l'équipe est dédiée, la question de l'affectation des ressources et de la visibilité n'est pas pour autant résolue. Dans l'entreprise A, l'équipe est dédiée mais les six personnes de l'équipe ne sont pas toutes rattachées à la même entité du groupe. Le dispositif n'a pas un positionnement clair dans l'organisation, ce qui rend opaque toutes les décisions organisationnelles qui y sont liées. En outre, il semble 
que cette barrière soit parfois amplifiée par la complexité organisationnelle de certaines entreprises.

"J'ai la fonction de co-responsable de projet côté [entité 1], et ma collègue côté [entité 2]. On est en projet miroir. C'est un arrangement un petit peu complexe mais c'est comme ça. » (Entreprise A, co-responsable de la plateforme, mai 2014).

Il apparaît que les questions organisationnelles sont perçues comme les plus difficiles à résoudre lors de la mise en œuvre du CS. Les acteurs reconnaissent unanimement le poids souvent sous-estimé des ressources nécessaires pour entretenir des relations denses et étroites avec la foule, notamment dans le cadre d'activités créatives. Concernant les autres barrières organisationnelles (implication interne, dimension culturelle, intégration organisationnelle) elles semblent affecter davantage les quatre grandes entreprises que la PME (C). L'entreprise C s'étant développée grâce au CS, ses acteurs et son organisation ont été peu à peu façonnés par les relations permanentes entretenues avec sa communauté d'utilisateurs. A l'inverse, il semble que lorsque le dispositif est mis en œuvre dans un contexte réglé et structuré comme celui des grandes entreprises, les questions d'implication, de culture et de structure semblent plus problématiques.

\subsection{LA CAPACITE D'ABSORPTION : UN FREIN IMPORTANT A L'INTEGRATION DES CONNAISSANCES}

Qu'il s'agisse d'idées dans le cadre du CS d'activités créatives ou de solutions dans le cadre du CS de résolution de problèmes complexes, les contributions provenant de la foule sont porteuses de connaissances pour les entreprises. Nos cas mettent en évidence que le développement de capacités d'absorption semble nécessaire pour tirer profit des contributions de la foule. Lorsque l'entreprise ne parvient pas à comprendre les soumissions qui lui sont faites, elle peut ainsi se priver d'un potentiel de connaissance pour innover.

"C'est compliqué quand on n'a pas la connaissance technique [...] il faut premièrement comprendre la solution, comprendre la proposition qu'on vous fait. Il faut avoir des experts en face de la communauté sur les sujets qu'on a définis pour pouvoir comprendre les propositions faites. » (Entreprise D, Responsable de l'innovation, mars 2014). 
Cela peut également poser un problème de crédibilité vis-à-vis de la foule. Lors d'échanges avec des experts, le community manager de la plateforme de CS de l'entreprise E n'a par exemple pas toujours su comprendre certaines soumissions. Cela a contribué à rendre les relations avec la foule plus distantes, puisque ce dernier, en prenant la parole au nom de l'entreprise, a mis en défaut l'image d'expert dont elle pouvait bénéficier. A l'inverse, le manque de maîtrise de certaines connaissances par la foule peut également constituer une limite et faire perdre du temps à l'entreprise.

"Celui qui ne sait pas comment c'est fait, il peut proposer des choses parfois fantaisistes » (Entreprise D, Dirigeant, juin 2014).

La barrière liée à l'absorption des connaissances semble affecter davantage le CS d'activités créatives. Dans le cas du CS de résolution de problèmes complexes, l'entreprise soumet à la foule un problème précis. Par ce ciblage, elle s'attend donc à recevoir des solutions spécifiques. Dans le cas du CS d'activités créatives, les entreprises ont moins de maitrise sur la nature des contributions reçues.

«Avec les candidatures spontanées, on ne sait pas à quoi s'attendre, ça peut partir dans tous les sens...Ça prend plus de temps pour évaluer l'inattendu. »(Entreprise B, ancien coresponsable de la plateforme, octobre 2015).

Les activités de CS de résolution de problèmes semblent moins soumises aux difficultés liées à l'absorption des connaissances, et ce pour plusieurs raisons. Tout d'abord, en ciblant l'appel, l'entreprise peut plus facilement contrôler le contenu des contributions attendues. Elle peut ainsi prévoir plus facilement les ressources et processus nécessaires au traitement et à l'évaluation de ces contributions. L'entreprise B explique par exemple qu'en fonction de la nature du problème à résoudre, des évaluateurs sont définis à l'avance en fonction de leur domaine d'expertise.

"L'intérêt pour les challenges, c'est que les évaluateurs sont les mêmes jusqu'à la date de clôture, pour tout ce qu'on reçoit. L'évaluation est plus simple que pour les candidatures spontanées » (Entreprise B, ancien co-responsable de la plateforme, octobre 2015). 
Par ailleurs, nos cas révèlent que l'ouverture de challenges de résolution de problèmes émane généralement d'un client interne (souvent issu de la $R \& D$ ) qui assure ensuite la sélection, le suivi et l'intégration des contributions. Cette conception décentralisée de l'usage du CS contribue sans doute à favoriser son adoption.

"Nos clients internes nous passent une commande. On valide avec eux l'objectif mais le livrable après, c'est eux qui le gèrent. » (Entreprise A, co-responsable de la plateforme, mai 2014).

Tableau 4 - synthèse des barrières à la mise en ouvre des activités de CS pour innover

\begin{tabular}{|c|c|c|c|c|c|c|}
\hline \multicolumn{2}{|r|}{ Cas } & $\mathbf{A}$ & B & $\mathbf{E}$ & D & $\mathbf{C}$ \\
\hline \multicolumn{2}{|c|}{ Type de crowdsourcing } & \multicolumn{3}{|c|}{ CS de résolution de problèmes } & \multicolumn{2}{|c|}{ CS d'activités créatives } \\
\hline \multirow{2}{*}{$\begin{array}{c}\text { Barrières } \\
\text { stratégiques (BS) }\end{array}$} & $\begin{array}{l}\text { Révélation d'informations } \\
\qquad(\mathrm{BS} 1)^{1}\end{array}$ & & $* *$ & $* *$ & & \\
\hline & $\begin{array}{l}\text { Manque de ressources } \\
\text { financières (BS2) }\end{array}$ & $*$ & $*$ & $*$ & $*$ & \\
\hline \multirow{4}{*}{$\begin{array}{c}\text { Barrières } \\
\text { organisationnelles } \\
\text { (BO) }\end{array}$} & $\begin{array}{l}\text { Culture, syndrome NIH } \\
\text { (BO3) }\end{array}$ & & $* * *$ & $* *$ & $*$ & \\
\hline & $\begin{array}{l}\text { Manque d'implication des } \\
\text { fonctions internes (BO4) }\end{array}$ & $*$ & $* * *$ & & $*$ & \\
\hline & $\begin{array}{l}\text { Coordination avec la foule } \\
\text { (BO5) }\end{array}$ & $*$ & $*$ & $* * *$ & $* * *$ & $* * *$ \\
\hline & $\begin{array}{l}\text { Intégration du CS à } \\
\text { l'organisation (BO6) }\end{array}$ & $* *$ & $*$ & $*$ & $* *$ & \\
\hline $\begin{array}{l}\text { Barrière cognitive } \\
\text { (BC) }\end{array}$ & $\begin{array}{c}\text { Capacité d'absorption } \\
\text { (BC7) }\end{array}$ & $*$ & $*$ & $*$ & $* *$ & $* *$ \\
\hline
\end{tabular}

$1:$ les cellules grisées désignent les barrières du CS identifiées au cours de nos entretiens. Les autres barrières sont celles identifiées dans la littérature. Les * correspondent au nombre de fois où chaque barrière a été évoquée par un verbatim pour chaque cas : * (barrière citée 1 à 5 fois) ** (barrière citée 5 à 10 fois) $* * *$ (barrière citée 10 à 15 fois). Cette catégorisation nous permet de repérer le poids relatif de chaque barrière qui vient corroborer le discours des acteurs sur l'importance des barrières.

\section{DISCUSSION ET CONCLUSION}

L'objectif de cet article est d'identifier les barrières à la mise en œuvre du CS pour innover.

Les cinq études de cas exploratoires nous permettent de dégager sept barrières pouvant se décliner en trois grandes catégories préexistantes proposées par la littérature (stratégique, organisationnelle et cognitive). Plusieurs contributions se dégagent de cette recherche.

Tout d'abord, alors que les rares études existantes portent pour l'essentiel sur la première phase d'initialisation du processus d'adoption de cette nouvelle activité (Di Gangi et Wasko, 
2009 ; Thuan et al., 2015), cette recherche apporte des éléments de réponse sur la deuxième phase plus critique de mise en usage (Damanpour et Schneider, 2006). En outre, elle porte sur l'ensemble des pratiques de CS pour innover et complète ainsi les travaux antérieurs focalisés sur des aspects plus ciblés. L'étude de Simula et Vuori (2012) sur les barrières à l'adoption du CS dans le contexte spécifique des relations BtoB offre une vision partielle des contraintes de gestion des relations directes potentiellement plus volumineuses et hétérogènes en BtoC étudiées ici. Lüttgens et al. (2014) se focalisent pour leur part sur les barrières du CS de résolution de problèmes et soulignent l'importance de barrières internes plus communément identifiées dans la littérature portant sur les barrières à l'adoption de nouvelles pratiques (Dubouloz, 2013). Nos résultats vont dans le sens de ces premiers travaux et les complètent en identifiant de nouvelles barrières absentes dans la littérature du CS : la barrière stratégique, liée à la révélation d'information, et les barrières organisationnelles, liées à la coordination avec la foule et à l'intégration du CS à l'organisation. Nous mettons plus particulièrement en évidence le rôle critique des barrières liées à la coordination avec la foule et à l'absorption des connaissances lors de cette phase de mise en usage du CS. Nous soulignons enfin que les barrières au CS semblent plus importantes pour le CS d'activités créatives, pour lesquelles la nature plus ouverte des contributions de la foule semble d'autant plus confronter l'entreprise à des barrières organisationnelles, comme celle de devoir gérer des suggestions nombreuses et hétérogènes (Piezunka et Dahlander, 2015).

L'augmentation des barrières dans le cas de la mise en œuvre du CS d'activités créatives questionne de manière plus fondamentale les relations entre l'entreprise et la foule. Alors que le CS de résolution de problèmes sous-entend des relations ponctuelles avec la foule (Pénin et Burger-Helmchen, 2012), le CS d'activités créatives requiert des échanges réguliers sur des périodes plus longues, rendant ainsi la coordination avec la foule plus difficile pour les entreprises de notre échantillon. Toutefois, ces échanges ne sont pas toujours à destination de 
la foule. Lorsqu'elle co-développe des innovations avec des pratiquants, l'entreprise C décrit en réalité des interactions avec une communauté (Dahlander et Frederiksen, 2012), c'est-àdire des individus qui échangent volontairement et régulièrement sur un sujet d'intérêt ou autour d'un objectif commun (Amin et Cohendet, 2004), et non pas avec une foule distante et anonyme (Schenk et Guittard, 2012). Cette entreprise cherche à attirer des individus passionnés par son cœur de métier au sein d'une communauté qu'elle rassemble virtuellement autour des activités de CS, appuyées par l'animation d'un forum virtuel, et plus directement par l'organisation d'activités sportives ou de moments de convivialité. Les cas de notre échantillon portant sur des activités créatives suggèrent ainsi que l'entreprise doit dépasser le cadre strict du CS et passer de relations ponctuelles et anonymes à des relations régulières avec des individus identifiés et le plus souvent regroupés en communauté.

Ce passage du crowdsourcing à ce que l'on pourrait appeler le communitysourcing pour les activités créatives peut s'expliquer de deux manières. En premier lieu, on peut songer à un phénomène de transformation de la foule. Au fur et à mesure des interactions, les individus les plus actifs de la foule sont sélectionnés et se structurent peu à peu pour constituer une communauté orientée sur les problématiques de l'entreprise (Parmentier, 2015). En second lieu, il est possible qu'une communauté préexiste indépendamment du CS et qu'en développant les relations avec ses membres, la communauté s'impose à l'entreprise. Sous certaines conditions, l'entreprise peut l'orienter et tirer parti de ses contributions (Jeppesen et Frederiksen, 2006). Bien que l'interaction avec une communauté conduise manifestement l'entreprise C à faire face à d'autres barrières organisationnelles (engagement dans des dispositifs organisationnels contraignants, augmentation des coûts de coordination liés au pilotage de la communauté etc.), il semble toutefois qu'elle limite ces types de barrières grâce à des relations régulières et approfondies avec les membres de sa communauté et à une délégation partielle de la coordination de la foule aux membres les plus actifs. Aussi, dans la 
lignée de Linder et al. (2003) et Simula et Ahola (2014), nos résultats suggèrent que dans certains contextes, tel le CS d'activités créatives, les entreprises devraient encourager le développement de communautés ou s'adresser à ces dernières et ainsi mettre en œuvre des activités de communitysourcing. Ce résultat va dans le sens des travaux de Blohm et al. (2013), qui suggèrent aux entreprises de développer des capacités de community building lorsqu'elles mettent en œuvre le CS.

Le développement de telles capacités par les entreprises pose toutefois la question des caractéristiques contextuelles de ces dernières, notamment en ce qui concerne leur taille et leur stratégie. Alors que Pénin et Burger-Helmchen (2012) ont souligné que la mise en œuvre du CS pour innover peut poser problème aux PME, nos cas semblent à l'inverse indiquer que les barrières perçues comme les plus problématiques pèsent davantage dans le contexte plus contraint et réglé des grandes entreprises que dans celui de la PME. En étant plus adaptable et plus agile que les premières (Kumar et al., 2012), l'entreprise C ne se sent pas affectée par les barrières stratégiques identifiées, à la différence des grandes entreprises qui doivent trouver des solutions pour les contourner. Par exemple, l'épineuse question de la confidentialité des informations mises en ligne n'est pas perçue de la même manière par l'entreprise C et par les autres entreprises. Son agilité lui permet notamment de s'affranchir de processus trop contraints et ainsi d'agir plus rapidement que ses concurrents, composés essentiellement de grandes entreprises. Au final, pour l'entreprise C, la révélation d'informations stratégiques n'est pas perçue comme un risque puisqu'elle pense innover plus rapidement que ses concurrents. De même, la question de l'intégration du CS au sein de l'organisation ne se pose pas pour l'entreprise C qui, bien qu'elle fasse des réajustements réguliers, a intégré le CS dans ses processus d'innovation dès sa création. Il semble ainsi que les barrières organisationnelles et stratégiques à la mise en œuvre du CS pour innover soient plus difficiles à franchir pour les grandes entreprises, où contrairement à l'entreprise $\mathrm{C}$, les projets de CS pour innover sont 
dédiés et situés en dehors des projets d'innovation. Or, dans le mesure où ce découplage peut nuire à la mise en œuvre de ces nouvelles pratiques ouvertes, nos résultats suggèrent plutôt de développer une démarche de CS au sein même des projets d'innovation.

D'un point de vue stratégique, nos résultats suggèrent également, à l'inverse de la littérature (Renault, 2014), que la propriété intellectuelle n'est pas perçue comme une barrière à la mise en œuvre du CS. En effet, même s'il s'agit d'un point important pour l'ensemble des répondants, cette dimension n'est pas assimilée à un obstacle. La question de la propriété intellectuelle est traitée en amont de la contribution de la foule, les individus étant par exemple tenus d'accepter de céder leurs droits avant de pouvoir participer. Ce résultat est comparable aux pratiques d'innovation ouverte identifiées dans le secteur des jeux-vidéo (Gandia et al., 2011). Cette littérature montre que les joueurs qui souhaitent créer du contenu sur des plateformes collaboratives ouvertes doivent se soumettre aux conditions contractuelles imposées par les entreprises du secteur qui les obligent à céder leurs droits de propriété pour pouvoir partager leurs contributions et ajouts auprès de la communauté de joueurs. En revanche, au-delà de la propriété intellectuelle, le risque de révéler des informations stratégiques se pose pour les grandes entreprises de notre échantillon. Si certaines ont trouvé des solutions pour atténuer cette barrière, comme l'utilisation d'une plateforme d'intermédiation (Schenk et Guittard, 2012), nos résultats indiquent que ce type de risques peut remettre en question la mise en usage du CS pour innover qui, à la différence du CS de tâches simples, porte sur des activités stratégiques (Brabham, 2008).

Cette recherche n'est pas exempte de limites. Tout d'abord, le nombre de cas nous invite à une certaine prudence quant à la portée des résultats de cette étude. En outre, contrairement à Lüttgens et al. (2014), nous nous sommes plus particulièrement intéressés à la phase de mise en œuvre du processus d'adoption. Or, à la lumière des résultats de ces auteurs, il semblerait que certaines barrières soient susceptibles d'apparaitre au début du processus et disparaitre 
par la suite, soit parce qu'elles ne concernent que certaines phases, soit parce que l'entreprise a été en mesure de les faire disparaître. Compte tenu de l'importance du rôle de l'organisation et de certains acteurs clés (Lüttgens et al., 2014) pour dépasser ces barrières, il apparaît pertinent d'étudier plus en profondeur les processus d'adoption du CS pour comprendre comment chaque type s'intègre ou complète les processus d'innovation des entreprises et étudier ainsi d'éventuels effets d'apprentissage (Pénin et Burger-Helmchen, 2012 ; Schlagwein et Bjørn-Andersen, 2014) sur l'intégralité du processus d'adoption d'une activité de CS pour innover. Une extension de ce travail exploratoire auprès d'un nombre plus important de cas couvrant l'ensemble du processus d'adoption des activités de CS pour innover ainsi qu'une plus grande variété d'acteurs interrogés par cas permettrait de répondre à ces limites.

\section{Références}

Afuah A. et Tucci C.L. (2012). «Crowdsourcing As a Solution to Distant Search », Academy of Management Review, vol. 37, $\mathrm{n}^{\circ}$ 3, 355-375.

Amin A. et Cohendet P. (2004). Architectures of knowledge: Firms, capabilities, and communities, Oxford University Press.

Blohm I., Leimeister J.M. et Krcmar H. (2013). « Crowdsourcing: How to Benefit from (Too) Many Great Ideas », MIS Quarterly Executive, vol. 12, n 4, 199-211.

Boutigny E. et Renault S. (2013). «Le Jam : analyse et enjeux de l'outil crowdsourcing d'IBM », Revue française de gestion, vol. 39, n² 236, 49-66.

Brabham D.C. (2008). «Crowdsourcing as a Model for Problem Solving An Introduction and Cases ». Convergence: The International Journal of Research into New Media Technologies, vol. 14, $\mathrm{n}^{\circ}$ 1, 75-90.

Busarovs A. (2011). «Crowdsourcing as User-Driven Innovation, New Business Philosophy's Model », Journal of Business Management, vol. 4, 53-60.

Chesbrough H.W. (2003). Open innovation: The new imperative for creating and profiting from technology, Harvard Business Press.

Cohen W.M. et Levinthal D.A. (1990). « Absorptive capacity: a new perspective on learning and innovation », Administrative science quarterly, vol. $35, \mathrm{n}^{\circ} 1,1128-1152$.

Dahlander L. et Frederiksen L. (2012). «The core and cosmopolitans: A relational view of innovation in user communities », Organization Science, vol. 23, n 4, 988-1007.

Damanpour F. (1991). «Organizational Innovation: A Meta-Analysis of Effects of Determinants and Moderators », Academy of Management Journal, vol. 34, n 3, 555-590. 
Damanpour F. et Schneider M. (2006). " Phases of the Adoption of Innovation in Organizations: Effects of Environment, Organization and Top Managers », British Journal of Management, vol. 17, $\mathrm{n}^{\circ}$ 3, 215-236.

Di Gangi P.M. et Wasko M. (2009). «Steal my idea! Organizational adoption of user innovations from a user innovation community: A case study of Dell IdeaStorm », Decision Support Systems, vol. 48, $\mathrm{n}^{\circ}$ 1, 303-312.

Di Guardo M.C. et Castriotta M. (2013). "The Challenge and Opportunities of Crowdsourcing Web Communities: An Italian Case Study », International Journal of Electronic Commerce Studies, vol. 4, n 1, 79-91.

Dubouloz S. (2013). «Les barrières à l'innovation organisationnelle : Le cas du Lean Management », Management international, vol. 17, $\mathrm{n}^{\circ}$ 4, 121-144.

Gandia R., Brion S. et Mothe C. (2011). «Innovation ouverte et management de la propriété intellectuelle », Revue française de gestion, vol. 210, $\mathrm{n}^{\circ}$ 1, 117-131.

Howe J. (2006). « The rise of crowdsourcing », Wired magazine, vol. 14, n 6, 1-4.

Jeppesen L.B. et Frederiksen L. (2006). «Why Do Users Contribute to Firm-Hosted User Communities? The Case of Computer-Controlled Music Instruments », Organization Science, vol. 17, $\mathrm{n}^{\circ} 1,45-63$.

Kumar K., Boesso G., Favotto F. et Menini A. (2012). «Strategic orientation, innovation patterns and performances of SMEs and large companies », Journal of Small Business and Enterprise Development, vol. 19, $\mathrm{n}^{\circ} 1,132-145$.

Lebraty J.F. (2009). «Externalisation ouverte et pérennité », Revue française de gestion, vol. $192, \mathrm{n}^{\circ} 2,151-165$.

Linder J.C., Jarvenpaa S. et Davenport T.H. (2003). «Toward an Innovation Sourcing Strategy », MIT Sloan Management Review, vol. 44, n 4, 43-49.

Lüttgens D., Pollok P., Antons D. et Piller F. (2014). «Wisdom of the Crowd and Capabilities of a Few: Internal Success Factors of Crowdsourcing for Innovation », Journal of Business Economics, vol. 84, $\mathrm{n}^{\circ}$ 3, 339-374.

March J.G. (1991). «Exploration and exploitation in organizational learning », Organization science, vol. $2, \mathrm{n}^{\circ} 1,71-87$.

Miles M.B. et Huberman A.M. (2003). Analyse des données qualitatives. De Boeck Supérieur.

Parmentier G. (2015). « How to Innovate with a Brand Community », Journal of Engineering \& Technology Management, vol. 37, 78-89.

Pénin J. et Burger-Helmchen T. (2012). «Crowdsourcing d'activités inventives et frontières des organisations », Management international, vol. 16, 101-112.

Piezunka H. et Dahlander L. (2015). « Distant search, narrow attention: how crowding alters organizations'filtering of suggestions in crowdsourcing », Academy of Management Journal, vol. 58, $\mathrm{n}^{\circ} 3,856-880$.

Renault S. (2014). «Crowdsourcing compétitif : ressorts et enjeux », Recherches en Sciences de Gestion, vol. 101, $\mathrm{n}^{\circ}$ 2, 59-80.

Santos F.M. et Eisenhardt K.M. (2005). «Organizational boundaries and theories of organization », Organization science, vol. 16, n 5, 491-508. 
Schenk E. et Guittard C. (2012). «Une typologie des pratiques de crowdsourcing: l'externalisation vers la foule, au-delà du processus d'innovation », Management international, vol. 16, 89-100.

Schlagwein D. et Bjørn-Andersen N. (2014), « Organizational Learning with Crowdsourcing: The Revelatory Case of LEGO », Journal of the Association for Information Systems, vol. $15, \mathrm{n}^{\circ} 11,754-778$.

Simula H. et Vuori M. (2012). «Benefits and Barriers of Crowdsourcing in B2b Firms: Generating Ideas with Internal and External Crowds », International Journal of Innovation Management, vol. 16, $\mathrm{n}^{\circ}$ 6, 1-19.

Simula H. et Ahola T. (2014). «A network perspective on idea and innovation crowdsourcing in industrial firms », Industrial Marketing Management, vol. 43, $\mathrm{n}^{\circ}$ 3, 400-408.

Thuan N.H., Antunes P. et Johnstone D. (2015). «Factors influencing the decision to crowdsource: A systematic literature review », Information Systems Frontiers, vol. 18, ${ }^{\circ}$ 1, 47-68.

von Hippel E. (1994). " "Sticky information" and the locus of problem solving: implications for innovation », Management science, vol. 40, $\mathrm{n}^{\circ} 4,429-439$.

von Hippel E. et Katz R. (2002). « Shifting Innovation to Users via Toolkits », Management Science, vol. 48, $\mathrm{n}^{\circ}$ 7, 821-833.

Yin R.K. (2013). Case study research: Design and methods. Sage publications. 